ANNALES

POLONICI MATHEMATICI

$93.2(2008)$

\title{
Some properties of and open problems on Hessian nilpotent polynomials
}

\author{
by Wenhua ZhaO (Normal, IL)
}

\begin{abstract}
In the recent work [BE1], [M], [Z1] and [Z2], the well-known Jacobian conjecture ([BCW], $[\mathrm{E}])$ has been reduced to a problem on $\mathrm{HN}$ (Hessian nilpotent) polynomials (the polynomials whose Hessian matrix is nilpotent) and their (deformed) inversion pairs. In this paper, we prove several results on HN polynomials, their (deformed) inversion pairs as well as on the associated symmetric polynomial or formal maps. We also propose some open problems for further study.
\end{abstract}

1. Introduction. In the recent work [BE1], [M], [Z1] and [Z2], the wellknown Jacobian conjecture (see $[\mathrm{BCW}]$ and $[\mathrm{E}]$ ) has been reduced to a problem on HN (Hessian nilpotent) polynomials, i.e. the polynomials whose Hessian matrix is nilpotent, and their (deformed) inversion pairs. In this paper, we prove some properties of HN polynomials, the (deformed) inversion pairs of HN polynomials, the associated symmetric polynomial or formal maps, and the graphs assigned to homogeneous harmonic polynomials. Another purpose of this paper is to draw the reader's attention to some open problems which we believe will be interesting and important for further study of these objects.

In this section we first discuss some background and motivation for the study of HN polynomials and their (deformed) inversion pairs. We also fix some terminology and notation that will be used throughout this paper. Then we describe the arrangement of the paper.

1.1. Background and motivation. Let $z=\left(z_{1}, \ldots, z_{n}\right)$ be $n$ free commutative variables. We denote by $\mathbb{C}[z]$ (resp. $\mathbb{C}[[z]]$ ) the algebra of polynomials (resp. formal power series) of $z$ over $\mathbb{C}$. A polynomial or formal power series $P(z)$ is said to be $H N$ (Hessian nilpotent) if its Hessian matrix Hes $P:=\left(\partial^{2} P / \partial z_{i} \partial z_{j}\right)$ is nilpotent. The study of HN polynomials is mainly

2000 Mathematics Subject Classification: 14R15, 32H02, 32A50.

Key words and phrases: Hessian nilpotent polynomials, inversion pairs, harmonic polynomials, Jacobian conjecture.

The author has been partially supported by NSA Grant R1-07-0053. 
motivated by the recent progress achieved in [BE1], $[\mathrm{M}]$ and $[\mathrm{Z} 2]$ on the well-known Jacobian conjecture (JC), which we will briefly explain below.

Recall that the JC first proposed by Keller [Ke] in 1939 claims: for any polynomial map $F$ of $\mathbb{C}^{n}$ with Jacobian $j(F)=1$, its formal inverse map $G$ is also a polynomial map. Despite intense study for more than half a century, the conjecture is still open even for the case $n=2$. For more history and known results before 2000 on the Jacobian conjecture, see [BCW], [E] and references there. In 2003, M. de Bondt and A. van den Essen ([BE1]) and G. Meng $([\mathrm{M}])$ independently made the following breakthrough on the JC.

Let $D_{i}:=\partial / \partial z_{i}(1 \leq i \leq n)$ and $D=\left(D_{1}, \ldots, D_{n}\right)$. For any $P(z) \in$ $\mathbb{C}[[z]]$, denote by $\nabla P(z)$ the gradient of $P(z)$, i.e.

$$
\nabla P(z):=\left(D_{1} P(z), \ldots, D_{n} P(z)\right) .
$$

We say a formal map $F(z)=z-H(z)$ is symmetric if $H(z)=\nabla P(z)$ for some $P(z) \in \mathbb{C}[[z]]$. Then the symmetric reduction of the JC achieved in $[\mathrm{BE} 1]$ and $[\mathrm{M}]$ is that to prove or disprove the $J C$, it is enough to consider only symmetric polynomial maps. Combining this with the classical homogeneous reduction achieved in $[\mathrm{BCW}]$ and $[\mathrm{Y}]$, one may further assume that the symmetric polynomial maps have the form $F(z)=z-\nabla P(z)$ with $P(z)$ homogeneous (of degree 4). Note that in this case the Jacobian condition $j(F)=1$ is equivalent to the condition that $P(z)$ is HN. For some other recent results on symmetric polynomial or formal maps, see [BE1]-[BE5], [EW], [M], [Wr1], [Wr2], [Z1], [Z2] and [EZ].

Based on the homogeneous reduction and the symmetric reduction of the JC discussed above, the author showed in [Z2] that the JC is actually equivalent to the following vanishing conjecture for $\mathrm{HN}$ polynomials.

Conjecture 1.1 (Vanishing Conjecture). Let $\Delta:=\sum_{i=1}^{n} D_{i}^{2}$ be the Laplace operator on $\mathbb{C}[z]$. Then, for any $H N$ polynomial $P(z)$ (of homogeneous of degree $d=4), \Delta^{m} P^{m+1}(z)=0$ when $m \gg 0$.

Furthermore, the following criterion of Hessian nilpotency for formal power series was also proved in [Z2].

Proposition 1.2. For any $P(z) \in \mathbb{C}[[z]]$ with $o(P(z)) \geq 2$, the following statements are equivalent.

(1) $P(z)$ is $H N$.

(2) $\Delta^{m} P^{m}=0$ for any $m \geq 1$.

(3) $\Delta^{m} P^{m}=0$ for any $1 \leq m \leq n$.

A crucial idea in [Z2] is to study a special formal deformation of symmetric formal maps. More precisely, let $t$ be a central formal parameter. For any $P(z) \in \mathbb{C}[[z]]$, we call $F(z)=z-\nabla P(z)$ the associated symmetric map of $P(z)$. Let $F_{t}(z)=z-t \nabla P(z)$. If the order $o(P(z))$ of $P(z)$ with respect 
to $z$ is greater than or equal to 2 , then $F_{t}(z)$ is a formal map of $\mathbb{C}[[t]][[z]]$ with $F_{t=1}(z)=F(z)$. Therefore, we may view $F_{t}(z)$ as a formal deformation of the formal map $F(z)$. In this case, one can also show (see $[\mathrm{M}]$ or Lemma 3.14 in [Z1]) that the formal inverse map $G_{t}(z):=F_{t}^{-1}(z)$ of $F_{t}(z)$ exists and is also symmetric, i.e. there exists a unique $Q_{t}(z) \in \mathbb{C}[[t]][[z]]$ with $o\left(Q_{t}(z)\right) \geq 2$ such that $G_{t}(z)=z+t \nabla Q_{t}(z)$. We call $Q_{t}(z)$ the deformed inversion pair of $P(z)$. Note that, whenever $Q_{t=1}(z)$ makes sense, the formal inverse map $G(z)$ of $F(z)$ is given by $G(z)=G_{t=1}(z)=z+\nabla Q_{t=1}(z)$, so in this case we call $Q(z):=Q_{t=1}(z)$ the inversion pair of $P(z)$.

Note that, under the condition $o(P(z)) \geq 2$, the deformed inversion pair $Q_{t}(z)$ of $P(z)$ might not be in $\mathbb{C}[t][[z]]$, so $Q_{t=1}(z)$ may not make sense. But if we assume further that $J\left(F_{t}\right)(0)=1$, or equivalently, $(\operatorname{Hes} P)(0)$ is nilpotent, then $F_{t}(z)$ is an automorphism of $\mathbb{C}[t][[z]]$, hence so is its inverse map $G_{t}(z)$. Therefore, in this case $Q_{t}(z)$ lies in $\mathbb{C}[t][[z]]$ and $Q_{t=1}(z)$ makes sense. Throughout this paper, whenever the inversion pair $Q(z)$ of a polynomial or formal power series $P(z) \in \mathbb{C}[[z]]$ (not necessarily HN) is considered, our assumption on $P(z)$ will always be $o(P(z)) \geq 2$ and $(\operatorname{Hes} P)(0)$ is nilpotent. Note that, for any HN $P(z) \in \mathbb{C}[[z]]$ with $o(P(z)) \geq 2$, the condition that $($ Hes $P)(0)$ is nilpotent holds automatically.

For later purposes, let us recall the following formula derived in [Z2] for the deformed inversion pairs of $\mathrm{HN}$ formal power series.

Theorem 1.3. Suppose $P(z) \in \mathbb{C}[[z]]$ with $o(P(z)) \geq 2$ is $H N$. Then

$$
Q_{t}(z)=\sum_{m=0}^{\infty} \frac{t^{m}}{2^{m} m !(m+1) !} \Delta^{m} P^{m+1}(z) .
$$

From the equivalence of the JC and the VC discussed above, we see that the study of $\mathrm{HN}$ polynomials and their (deformed) inversion pairs becomes important and necessary, at least when the JC is concerned. Note that, due to the identity $\operatorname{Tr}$ Hes $P=\Delta P$, HN polynomials are just a special family of harmonic polynomials which are among the most classical objects in mathematics. Even though harmonic polynomials has been very well studied since the late eighteenth century, it seems that not much is known about HN polynomials. We believe that they deserve much more attention from mathematicians.

1.2. Arrangement. Considering the length of this paper, we here describe its arrangement in more detail.

In Section 2, we consider the following two questions. Let $P, S, T \in \mathbb{C}[[z]]$ with $P=S+T$, and $Q, U, V$ their respective inversion pairs.

$\mathbf{Q}_{1}$ : Under what conditions, $P$ is $H N$ iff both $S$ and $T$ are $H N$ ?

$\mathbf{Q}_{2}$ : Under what conditions, $Q=U+V$ ? 
We give some sufficient conditions in Theorems 2.1 and 2.7 for the two questions above. In Section 3, we employ a recursion formula for inversion pairs derived in [Z1] and (1.1) above to derive some estimates for the radius of convergence of inversion pairs of homogeneous HN polynomials (see Propositions 3.1 and 3.3).

We say that $P(z) \in \mathbb{C}[[z]]$ is self-inverting if its inversion pair $Q(z)$ is $P(z)$ itself. In Section 4, by using a general result on quasi-translations proved in $[\mathrm{B}]$, we derive some properties of $\mathrm{HN}$ self-inverting formal power series. Another purpose of that section is to draw the reader's attention to Open Problem 4.8 on classification of HN self-inverting polynomials or formal power series.

In Section 5, we show in Proposition 5.1 that when the base field has characteristic $p>0$, the $\mathrm{VC}$, unlike the JC, actually holds for any polynomial $P(z)$ even without the $\mathrm{HN}$ condition on $P(z)$. It also holds in this case for any $\mathrm{HN}$ formal power series. One interesting question (see Open Problem 5.2) is to see if the VC like the JC fails over $\mathbb{C}$ when $P(z)$ is allowed to be any $\mathrm{HN}$ formal power series.

In Section 6, we prove a criterion of Hessian nilpotency for homogeneous polynomials over $\mathbb{C}$ (see Theorem 6.1). Considering the criterion in Proposition 1.2, this criterion is somewhat surprising but its proof turns out to be very simple.

Section 7 is mainly motivated by the following question raised by M. Kumar $([\mathrm{K}])$ and D. Wright ([Wr3]): for a symmetric formal map $F(z)=$ $z-\nabla P(z)$, how to write $f(z):=\frac{1}{2} \sigma_{2}-P(z)$ (where $\sigma_{2}:=\sum_{i=1}^{n} z_{i}^{2}$ ) and $P(z)$ itself as formal power series in $F(z)$ ? In that section, we derive some explicit formulas to answer this question and also the same question for $\sigma_{2}$ (see Proposition 7.2). From those formulas, we also show in Theorem 7.4 that the VC holds for a HN polynomial $P(z)$ iff one (hence, all) of $\sigma_{2}, P(z)$ and $f(z)$ can be written as a polynomial in $F$, where $F(z)=z-\nabla P(z)$ is the associated polynomial map of $P(z)$.

Finally, in Section 8, we discuss a graph $\mathcal{G}(P)$ assigned to each homogeneous harmonic polynomial $P(z)$. The graph $\mathcal{G}(P)$ was first proposed by the author and later studied by Roel Willems in his master thesis [Wi] under the direction of Arno van den Essen. In Subsection 8.1 we give the definition of $\mathcal{G}(P)$ for any homogeneous harmonic polynomial $P(z)$ and discuss the connectedness reduction (see Corollary 8.5) which says that to study the VC for homogeneous HN polynomials $P(z)$, it is enough to consider the case when the graph $\mathcal{G}(P)$ is connected. In Subsection 8.2 we consider a connection of $\mathcal{G}(P)$ with the tree expansion formula derived in $[\mathrm{M}]$ and $[\mathrm{Wr} 2]$ for the inversion pair $Q(z)$ of $P(z)$ (see also Proposition 8.9). As an application, we give another proof for the connectedness reduction discussed in Corollary 8.5. 
Finally, we remark that even though we could have focused only on HN polynomials, at least when only the $\mathrm{JC}$ is concerned, we formulate and prove our results in the more general setting of $\mathrm{HN}$ formal power series whenever possible.

Acknowledgements. The author is grateful to Professors Arno van den Essen, Mohan Kumar and David Wright for inspiring communications and constant encouragement. Section 7 was mainly motivated by some questions raised by Mohan Kumar and David Wright. The author would also like to thank Roel Willems for sending his master thesis in which he has obtained some very interesting results on the graphs $\mathcal{G}(P)$ of homogeneous harmonic polynomials. Last but not least, the author thanks the referee and the editor for many valuable suggestions.

2. Disjoint formal power series and their deformed inversion pairs. Let $P, S, T \in \mathbb{C}[[z]]$ with $P=S+T$, and $Q, U, V$ their respective inversion pairs. In this section, we consider the following two questions:

$\mathbf{Q}_{1}$ : Under what conditions, $P$ is $H N$ if and only if both $S$ and $T$ are $H N$ ?

$\mathbf{Q}_{2}$ : Under what conditions, $Q=U+V$ ?

We give some answers to questions $\mathbf{Q}_{1}$ and $\mathbf{Q}_{2}$ in Theorems 2.1 and 2.7, respectively. The results proved here will also be needed in Section 8 when we consider a graph associated to homogeneous harmonic polynomials.

For question $\mathbf{Q}_{1}$ above, we have the following result.

Theorem 2.1. Let $S, T \in \mathbb{C}[[z]]$ be such that $\left\langle\nabla\left(D_{i} S\right), \nabla\left(D_{j} T\right)\right\rangle=0$ for any $1 \leq i, j \leq n$, where $\langle\cdot, \cdot\rangle$ denotes the standard $\mathbb{C}$-bilinear form on $\mathbb{C}^{n}$. Let $P=S+T$. Then:

(a) $\operatorname{Hes}(S) \operatorname{Hes}(T)=\operatorname{Hes}(T) \operatorname{Hes}(S)=0$.

(b) $P$ is $H N$ iff both $S$ and $T$ are $H N$.

Note that statement (b) was first proved by R. Willems ([Wi]) in a special setting as in Lemma 2.6 below for homogeneous harmonic polynomials.

Proof. (a) For any $1 \leq i, j \leq n$, consider the $(i, j)$ th entry of the product $\operatorname{Hes}(S) \operatorname{Hes}(T)$ :

$$
\sum_{k=1}^{n} \frac{\partial^{2} S}{\partial z_{i} \partial z_{k}} \frac{\partial^{2} T}{\partial z_{k} \partial z_{j}}=\left\langle\nabla\left(D_{i} S\right), \nabla\left(D_{j} T\right)\right\rangle=0 .
$$

Hence $\operatorname{Hes}(S) \operatorname{Hes}(T)=0$. Similarly, we have $\operatorname{Hes}(T) \operatorname{Hes}(S)=0$.

(b) follows directly from (a) and the lemma below.

Lemma 2.2. Let $A, B$ and $C$ be $n \times n$ matrices with entries in any commutative ring. Suppose that $A=B+C$ and $B C=C B=0$. Then $A$ is nilpotent iff both $B$ and $C$ are nilpotent. 
Proof. The $(\Leftarrow)$ part is trivial because $B$ and $C$ in particular commute with each other.

To show $(\Rightarrow)$, note that $B C=C B=0$. So for any $m \geq 1$, we have

$$
A^{m} B=(B+C)^{m} B=\left(B^{m}+C^{m}\right) B=B^{m+1} .
$$

Similarly, we have $C^{m+1}=A^{m} C$. Therefore, if $A^{N}=0$ for some $N \geq 1$, we have $B^{N+1}=C^{N+1}=0$.

Note that, for the $(\Leftarrow)$ part of $(\mathrm{b})$ in Theorem 2.1 , we only need a weaker condition: for any $1 \leq i, j \leq n$,

$$
\left\langle\nabla\left(D_{i} S\right), \nabla\left(D_{j} T\right)\right\rangle=\left\langle\nabla\left(D_{j} S\right), \nabla\left(D_{i} T\right)\right\rangle,
$$

which ensures that $\operatorname{Hes}(S)$ and $\operatorname{Hes}(T)$ commute.

To consider the second question $\mathbf{Q}_{2}$, let us first fix the following notation.

For any $P \in \mathbb{C}[[z]]$, let $\mathcal{A}(P)$ denote the subalgebra of $\mathbb{C}[[z]]$ generated by all partial derivatives of $P$ (of any order). We also define a sequence $\left\{Q_{[m]}(z) \mid m \geq 1\right\}$ by writing the deformed inversion pair $Q_{t}(z)$ of $P(z)$ as

$$
Q_{t}(z)=\sum_{m \geq 1} t^{m-1} Q_{[m]}(z) .
$$

Lemma 2.3. For any $P \in \mathbb{C}[[z]]$, we have:

(a) $\mathcal{A}(P)$ is closed under the action of any differential operator on $\mathbb{C}[z]$ with constant coefficients.

(b) For any $m \geq 1$, we have $Q_{[m]}(z) \in \mathcal{A}(P)$.

Proof. (a) Note that, by the definition of $\mathcal{A}(P)$, a formal power series $g(z) \in \mathbb{C}[[z]]$ lies in $\mathcal{A}(P)$ iff it can be written (not necessarily uniquely) as a polynomial in partial derivatives of $P(z)$. Then, by the Leibniz rule, it is easy to see that, for any $g(z) \in \mathcal{A}(P), D_{i} g(z) \in \mathcal{A}(P)(1 \leq i \leq n)$. Repeating this argument, we see that any partial derivative of $g(z)$ is in $\mathcal{A}(P)$. Hence (a) follows.

(b) Recall that, by Proposition 3.7 in [Z1], we have the following recurrent formula for $Q_{[m]}(z)(m \geq 1)$ in general:

$$
\begin{aligned}
Q_{[1]}(z) & =P(z), \\
Q_{[m]}(z) & =\frac{1}{2(m-1)} \sum_{\substack{k, l \geq 1 \\
k+l=m}}\left\langle\nabla Q_{[k]}(z), \nabla Q_{[l]}(z)\right\rangle \quad \text { for any } m \geq 2 .
\end{aligned}
$$

By using (a), the recurrent formulas above and induction on $m \geq 1$, it is easy to check that (b) holds.

Definition 2.4. For any $S, T \in \mathbb{C}[[z]]$, we say $S$ and $T$ are disjoint if, for any $g_{1} \in \mathcal{A}(S)$ and $g_{2} \in \mathcal{A}(T)$, we have $\left\langle\nabla g_{1}, \nabla g_{2}\right\rangle=0$. 
This terminology will be justified in Section 8 when we consider a graph $\mathcal{G}(P)$ associated to homogeneous harmonic polynomials $P$.

Lemma 2.5. Let $S, T \in \mathbb{C}[[z]]$. Then $S$ and $T$ are disjoint iff, for any $\alpha, \beta \in \mathbb{N}^{n}$, we have

$$
\left\langle\nabla\left(D^{\alpha} S\right), \nabla\left(D^{\beta} T\right)\right\rangle=0 .
$$

Proof. The $(\Rightarrow)$ part is trivial. Conversely, for any $g_{1} \in \mathcal{A}(S)$ and $g_{2} \in$ $\mathcal{A}(T)(i=1,2)$, we need to show

$$
\left\langle\nabla g_{1}, \nabla g_{2}\right\rangle=0 \text {. }
$$

But this can be easily checked by first reducing to the case where $g_{1}$ and $g_{2}$ are monomials in partial derivatives of $S$ and $T$, respectively, and then applying the Leibniz rule and (2.5).

Examples of disjoint polynomials or formal power series are given in the following lemma, which will also be needed later in Section 8.

Lemma 2.6. Let $I_{1}$ and $I_{2}$ be two finite subsets of $\mathbb{C}^{n}$ such that, for any $\alpha_{i} \in I_{i}(i=1,2)$, we have $\left\langle\alpha_{1}, \alpha_{2}\right\rangle=0$. Denote by $\mathcal{A}_{i}(i=1,2)$ the completion of the subalgebra of $\mathbb{C}[[z]]$ generated by $h_{\alpha}(z):=\langle\alpha, z\rangle\left(\alpha \in I_{i}\right)$, i.e. $\mathcal{A}_{i}$ is the set of all formal power series in $h_{\alpha}(z)\left(\alpha \in I_{i}\right)$ over $\mathbb{C}$. Then, for any $P_{i} \in \mathcal{A}_{i}(i=1,2), P_{1}$ and $P_{2}$ are disjoint.

Proof. First, by a similar argument to the proof of Lemma 2.3(a), it is easy to check that $\mathcal{A}_{i}(i=1,2)$ is closed under the action of any differential operator with constant coefficients. Secondly, since each $\mathcal{A}_{i}$ is a subalgebra of $\mathbb{C}[[z]]$, we have $\mathcal{A}\left(P_{i}\right) \subset \mathcal{A}_{i}(i=1,2)$.

Therefore, to show that $P_{1}$ and $P_{2}$ are disjoint, it will be enough to show that, for any $g_{i} \in \mathcal{A}_{i}(i=1,2)$, we have $\left\langle\nabla g_{1}, \nabla g_{2}\right\rangle=0$. But this can be easily checked by first reducing to the case when $g_{i}(i=1,2)$ are monomials in $h_{\alpha}(z)\left(\alpha \in I_{i}\right)$, and then applying the Leibniz rule and the following identity: for any $\alpha, \beta \in \mathbb{C}^{n}$,

$$
\left\langle\nabla h_{\alpha}(z), \nabla h_{\beta}(z)\right\rangle=\langle\alpha, \beta\rangle .
$$

Now, for question $\mathbf{Q}_{2}$, we have the following result.

Theorem 2.7. Let $P, S, T \in \mathbb{C}[[z]]$ with order at least 2 , and $Q_{t}, U_{t}, V_{t}$ their respective deformed inversion pairs. Assume that $P=S+T$ and $S, T$ are disjoint. Then:

(a) $U_{t}$ and $V_{t}$ are also disjoint, i.e. for any $\alpha, \beta \in \mathbb{N}^{n}$,

$$
\left\langle\nabla D^{\alpha} U_{t}(z), \nabla D^{\beta} V_{t}(z)\right\rangle=0 .
$$

(b) We further have

$$
Q_{t}=U_{t}+V_{t} .
$$


Proof. (a) follows directly from Lemmas 2.3(b) and 2.5.

(b) Let $Q_{[m]}, U_{[m]}$ and $V_{[m]}(m \geq 1)$ be defined as in (2.2). Hence it is enough to show

$$
Q_{[m]}=U_{[m]}+V_{[m]}
$$

for any $m \geq 1$. We use induction on $m \geq 1$. When $m=1$, (2.7) follows from the condition $P=S+T$ and (2.3). For any $m \geq 2$, by (2.4) and the induction assumption, we have

$$
\begin{aligned}
Q_{[m]} & =\frac{1}{2(m-1)} \sum_{\substack{k, l \geq 1 \\
k+l=m}}\left\langle\nabla Q_{[k]}, \nabla Q_{[l]}\right\rangle \\
& =\frac{1}{2(m-1)} \sum_{\substack{k, l \geq 1 \\
k+l=m}}\left\langle\nabla U_{[k]}+\nabla V_{[k]}, \nabla U_{[l]}+\nabla V_{[l]}\right\rangle
\end{aligned}
$$

(noting that, by Lemma $2.3, U_{[j]} \in \mathcal{A}(S)$ and $V_{[j]} \in \mathcal{A}(T), 1 \leq j \leq m$ :)

$$
=\frac{1}{2(m-1)} \sum_{\substack{k, l \geq 1 \\ k+l=m}}\left\langle\nabla U_{[k]}, \nabla U_{[l]}\right\rangle+\frac{1}{2(m-1)} \sum_{\substack{k, l \geq 1 \\ k+l=m}}\left\langle\nabla V_{[k]}, \nabla V_{[l]}\right\rangle
$$

(applying the recursion formula (2.4) to both $U_{[m]}$ and $V_{[m]}$ :)

$$
=U_{[m]}+V_{[m]}
$$

As will be pointed out later in Remark 8.11, one can also prove this theorem by using a tree expansion formula for inversion pairs, which was derived in $[\mathrm{M}]$ and $[\mathrm{Wr} 2]$, in the setting of Lemma 2.6.

From Theorems 2.1, 2.7 and (1.1), (2.2), it is easy to see that we have the following corollary.

Corollary 2.8. Let $P_{i} \in \mathbb{C}[[z]](1 \leq i \leq k)$ be pairwise disjoint. Set $P=\sum_{i=1}^{k} P_{i}$. Then:

(a) $P$ is $H N$ iff each $P_{i}$ is $H N$.

(b) Suppose that $P$ is $H N$. Then, for any $m \geq 0$,

$$
\Delta^{m} P^{m+1}=\sum_{i=1}^{k} \Delta^{m} P_{i}^{m+1} .
$$

Consequently, if the VC holds for each $P_{i}$, then it also holds for $P$.

3. Local convergence of deformed inversion pairs of homogeneous HN polynomials. Let $P(z)$ be a formal power series which is convergent near $0 \in \mathbb{C}^{n}$. Then the associated symmetric map $F(z)=z-\nabla P$ is a well-defined analytic map from an open neighborhood of $0 \in \mathbb{C}^{n}$ to $\mathbb{C}^{n}$. 
If we further assume that $J F(0)=I_{n \times n}$, then the formal inverse $G(z)=$ $z+\nabla Q(z)$ of $F(z)$ is also locally well-defined analytic map. So the inversion pair $Q(z)$ of $P(z)$ is also locally convergent near $0 \in \mathbb{C}^{n}$. In this section, we use formulas (2.4), (1.1) and the Cauchy estimates to derive some estimates for the radius of convergence of inversion pairs of homogeneous HN polynomials (see Propositions 3.1 and 3.3).

First let us fix the following notation.

For any $a \in \mathbb{C}^{n}$ and $r>0$, we denote by $B(a, r)$ (resp. $S(a, r)$ ) the open ball (resp. sphere) centered at $a \in \mathbb{C}$ with radius $r>0$. The unit sphere $S(0,1)$ will also be denoted by $S^{2 n-1}$. Furthermore, we let $\Omega(a, r)$ be the polydisk centered at $a \in \mathbb{C}^{n}$ with radius $r>0$, i.e. $\Omega(a, r):=\left\{z \in \mathbb{C}^{n} \mid\right.$ $\left.\left|z_{i}-a_{i}\right|<r, 1 \leq i \leq n\right\}$. For any subset $A \subset \mathbb{C}^{n}$, we will use $\bar{A}$ to denote the closure of $A$ in $\mathbb{C}^{n}$.

For any polynomial $P(z) \in \mathbb{C}[z]$ and a compact subset $D \subset \mathbb{C}^{n}$, we let $|P|_{D}$ be the maximum value of $|P(z)|$ over $D$. In particular, when $D$ is the unit sphere $S^{2 n-1}$, we also write $|P|=|P|_{D}$, i.e.

$$
|P|:=\max \left\{|P(z)| \mid z \in S^{2 n-1}\right\} .
$$

Note that, for any $r \geq 0$ and $a \in B(0, r)$, we have $\Omega(a, r) \subset B(a, r) \subset$ $B(0,2 r)$. Combining this with the well-known maximum principle for holomorphic functions, we get

$$
|P|_{\overline{\Omega(a, r)}} \leq|P|_{\overline{B(a, r)}} \leq|P|_{\overline{B(0,2 r)}}=|P|_{S(0,2 r)} .
$$

For the inversion pair $Q$ of a homogeneous polynomial $P$ without the $\mathrm{HN}$ condition, we have the following estimate for the radius of convergence at $0 \in \mathbb{C}^{n}$.

Proposition 3.1. Let $P(z)$ be a non-zero homogeneous polynomial (not necessarily $H N)$ of degree $d \geq 3$ and $r_{0}=\left(n 2^{d-1}|P|\right)^{1 /(2-d)}$. Then the inversion pair $Q(z)$ converges over the open ball $B\left(0, r_{0}\right)$.

To prove the proposition, we need the following lemma.

Lemma 3.2. Let $P(z)$ be any polynomial and $r>0$. Then, for any $a \in B(0, r)$ and $m \geq 1$, we have

$$
\left|Q_{[m]}(a)\right| \leq \frac{n^{m-1}|P|_{S(0,2 r)}^{m}}{2^{m-1} r^{2 m-2}} .
$$

Proof. We use induction on $m \geq 1$. First, when $m=1$, by (2.3) we have $Q_{[1]}=P$. Then (3.3) follows from the fact that $B(a, r) \subset B(0,2 r)$ and from the maximum principle for holomorphic functions. 
Assume (3.3) holds for any $1 \leq k \leq m-1$. Then, by the Cauchy estimates of holomorphic functions (see e.g. Theorem 1.6 in $[R]$ ), we have

$$
\left|\left(D_{i} Q_{[k]}\right)(a)\right| \leq \frac{1}{r}\left|Q_{[k]}\right|_{\overline{\Omega(0, r)}} \leq \frac{n^{k-1}|P|_{B(0,2 r)}^{k}}{2^{k-1} r^{2 k-1}} .
$$

By (2.4) and (3.4), we have

$$
\begin{aligned}
\left|Q_{[m]}(a)\right| & \leq \frac{1}{2(m-1)} \sum_{\substack{k, l \geq 1 \\
k+l=m}}\left|\left\langle\nabla Q_{[k]}, \nabla Q_{[l]}\right\rangle\right| \\
& \leq \frac{1}{2(m-1)} \sum_{\substack{k, l \geq 1 \\
k+l=m}} n \frac{n^{k-1}|P|_{S(0,2 r)}^{k}}{2^{k-1} r^{2 k-1}} \frac{n^{l-1}|P|_{S(0,2 r)}^{l}}{2^{l-1} r^{2 l-1}} \\
& =\frac{n^{m-1}|P|_{S(0,2 r)}^{m}}{2^{m-1} r^{2 m-2}}
\end{aligned}
$$

Proof of Proposition 3.1. By (2.2), we know that,

$$
Q(z)=\sum_{m \geq 1} Q_{[m]}(z)
$$

To show the proposition, it is enough to show that the infinite series above converges absolutely over $B(0, r)$ for any $r<r_{0}$.

First, for any $m \geq 1$, let $A_{m}$ be the RHS of (3.3). Note that, since $P$ is homogeneous of degree $d \geq 3$, we have

$$
|P|_{B(0,2 r)}^{m}=\left((2 r)^{d}|P|_{S^{2 n-1}}\right)^{m}=(2 r)^{d m}|P|^{m} .
$$

Therefore, for any $m \geq 1$,

$$
A_{m}=2^{(d-1) m+1} n^{m-1} r^{(d-2) m+2}|P|^{m},
$$

and by Lemma 3.2 ,

$$
\left|Q_{[m]}(a)\right| \leq A_{m} \quad \text { for any } a \in B(0, r) .
$$

Since $0<r<r_{0}=\left(n 2^{d-1}|P|\right)^{2-d}$, it is easy to see that

$$
\lim _{m \rightarrow \infty} \frac{A_{m+1}}{A_{m}}=n 2^{d-1} r^{d-2}|P|<1 .
$$

Therefore, by the comparison test, the series in (3.5) converges absolutely and uniformly over $B(0, r)$.

Note that the estimate in Proposition 3.1 depends on the number $n$ of variables. Next we show that, with the $\mathrm{HN}$ condition on $P$, an estimate independent of $n$ can be obtained as follows.

Proposition 3.3. Let $P(z)$ be a homogeneous $H N$ polynomial of degree $d \geq 4$ and set $r_{0}:=\left(2^{d+1}|P|\right)^{1 /(2-d)}$. Then the inversion pair $Q(z)$ of $P(z)$ converges over the open ball $B\left(0, r_{0}\right)$. 
Note that, when $d=2$ or 3 , by Wang's theorem ([Wa]), the JC holds in general. Hence it also holds for the associated symmetric map $F(z)=z-\nabla P$ when $P(z)$ is HN. Therefore $Q(z)$ in this case is also a polynomial of $z$ and converges over the whole space $\mathbb{C}^{n}$.

To prove the proposition above, we first need the following two lemmas.

LEMma 3.4. Let $P(z)$ be a homogeneous polynomial of degree $d \geq 1$ and $r>0$. For any $a \in B(0, r), m \geq 0$ and $\alpha \in \mathbb{N}^{n}$, we have

$$
\left|\left(D^{\alpha} P^{m+1}\right)(a)\right| \leq \frac{\alpha !}{r^{|\alpha|}}(2 r)^{d(m+1)}|P|^{m+1} .
$$

Proof. First, by the Cauchy estimates and (3.2), we have

$$
\left|\left(D^{\alpha} P^{m+1}\right)(a)\right| \leq \frac{\alpha !}{r^{|\alpha|}}\left|P^{m+1}\right|_{\overline{\Omega(a, r)}} \leq \frac{\alpha !}{r^{|\alpha|}}\left|P^{m+1}\right|_{\overline{B(0,2 r)}} .
$$

On the other hand, by the maximum principle and the condition that $P$ is homogeneous of degree $d \geq 3$, we have

$$
\begin{aligned}
\left|P^{m+1}\right| \frac{\overline{B(0,2 r)}}{} & =|P| \frac{m+1}{B(0,2 r)}=|P| \frac{m+1}{S(0,2 r)}=\left((2 r)^{d}|P|\right)^{m+1} \\
& =(2 r)^{d(m+1)}|P|^{m+1} .
\end{aligned}
$$

Then, combining (3.10) and (3.11), we get (3.9).

LEMma 3.5. For any $m \geq 1$, we have

$$
\sum_{\substack{\alpha \in \mathbb{N}^{n} \\
|\alpha|=m}} \alpha ! \leq m !\left(\begin{array}{c}
m+n-1 \\
m
\end{array}\right)=\frac{(m+n-1) !}{(n-1) !} .
$$

Proof. First, for any $\alpha \in \mathbb{N}^{n}$ with $|\alpha|=m$, we have $\alpha ! \leq m$ ! since $\left(\begin{array}{c}m \\ \alpha\end{array}\right)=\frac{m !}{\alpha !}$ is always a positive integer. Therefore,

$$
\sum_{\substack{\alpha \in \mathbb{N}^{n} \\|\alpha|=m}} \alpha ! \leq m ! \sum_{\substack{\alpha \in \mathbb{N}^{n} \\|\alpha|=m}} 1
$$

Secondly, $\sum_{\alpha \in \mathbb{N}^{n},|\alpha|=m} 1$ is just the number of distinct $\alpha \in \mathbb{N}^{n}$ with $|\alpha|=m$, which is the same as the number of distinct monomials in $n$ free commutative variables of degree $m$. Since the latter is well-known to be $\left(\begin{array}{c}m+n-1 \\ m\end{array}\right)$, we have

$$
\sum_{\substack{\alpha \in \mathbb{N}^{n} \\
|\alpha|=m}} \alpha ! \leq m !\left(\begin{array}{c}
m+n-1 \\
m
\end{array}\right)=\frac{(m+n-1) !}{(n-1) !} .
$$

Proof of Proposition 3.3. By (1.1), we know that

$$
Q(z)=\sum_{m \geq 1} \frac{\Delta^{m} P^{m+1}}{2^{m} m !(m+1) !} .
$$


To show the proposition, it is enough to show that the infinite series above converges absolutely over $B(0, r)$ for any $r<r_{0}$.

We first give an upper bound for the general term in (3.13) over $B(0, r)$. We have

$$
\Delta^{m} P^{m+1}=\left(\sum_{i=1}^{n} D_{i}^{2}\right)^{m} P^{m+1}=\sum_{\substack{\alpha \in \mathbb{N}^{n} \\|\alpha|=m}} \frac{m !}{\alpha !} D^{2 \alpha} P^{m+1} .
$$

Therefore,

$$
\left|\Delta^{m} P^{m+1}(a)\right| \leq \sum_{\substack{\alpha \in \mathbb{N}^{n} \\|\alpha|=m}} \frac{m !}{\alpha !}\left|D^{2 \alpha} P^{m+1}(a)\right|
$$

(applying Lemma 3.4 with $\alpha$ replaced by $2 \alpha$ :)

$$
\leq \sum_{\substack{\alpha \in \mathbb{N}^{n} \\|\alpha|=m}} \frac{m !}{\alpha !} \frac{(2 \alpha) !}{r^{2 m}}(2 r)^{d(m+1)}|P|^{m+1}
$$

(noting that $(2 \alpha) ! \leq[(2 \alpha) ! !]^{2}=2^{2 m}(\alpha !)^{2}$ :)

$$
\begin{aligned}
& \leq \sum_{\substack{\alpha \in \mathbb{N}^{n} \\
|\alpha|=m}} \frac{m !}{\alpha !} \frac{2^{2 m}(\alpha !)^{2}}{r^{2 m}}(2 r)^{d(m+1)}|P|^{m+1} \\
& =m ! 2^{2 m+d(m+1)} r^{d(m+1)-2 m}|P|^{m+1} \sum_{\substack{\alpha \in \mathbb{N}^{n} \\
|\alpha|=m}} \alpha !
\end{aligned}
$$

(applying Lemma 3.5:)

$$
=\frac{m !(m+n-1) ! 2^{2 m+d(m+1)} r^{d(m+1)-2 m}|P|^{m+1}}{(n-1) !} .
$$

Therefore, for any $m \geq 1$, we have

$$
\left|\frac{\Delta^{m} P^{m+1}}{2^{m} m !(m+1) !}\right| \leq \frac{2^{m+d(m+1)} r^{d(m+1)-2 m}|P|^{m+1}(m+n-1) !}{(m+1) !(n-1) !} .
$$

For any $m \geq 1$, let $A_{m}$ be the right hand side of (3.15). Then, by a straightforward calculation,

$$
\frac{A_{m+1}}{A_{m}}=\frac{m+n}{m+2} 2^{d+1} r^{d-2}|P| .
$$

Since $r<r_{0}=\left(2^{d+1}|P|\right)^{1 /(2-d)}$, it is easy to see that

$$
\lim _{m \rightarrow \infty} \frac{A_{m+1}}{A_{m}}=2^{d+1} r^{d-2}|P|<1 .
$$


Therefore, by the comparison test, the series in (3.13) converges absolutely and uniformly over $B(0, r)$.

4. Self-inverting formal power series. Note that, by the definition of inversion pair, $Q \in \mathbb{C}[[z]]$ is the inversion pair of $P \in \mathbb{C}[[z]]$ iff $P$ is the inversion pair of $Q$. In other words, the relation that $Q$ and $P$ are the inversion pairs of each other is in some sense a duality relation. Naturally, one may ask which $P(z)$ are self-dual or self-inverting In this section, we discuss this special family of polynomials or formal power series.

Another purpose of this section is to draw the reader's attention to the problem of classification of HN self-inverting polynomials (see Open Problem 4.8). Even though the classification of $\mathrm{HN}$ polynomials seems to be out of reach at present, we believe that the classification of (HN) self-inverting polynomials is much more approachable.

Definition 4.1. A formal power series $P(z) \in \mathbb{C}[[z]]$ with $o(P(z)) \geq 2$ and $(\operatorname{Hes} P)(0)$ nilpotent is said to be self-inverting if its inversion pair is $P(z)$.

Following the terminology introduced in [B], we say a formal map $F(z)=$ $z-H(z)$ with $H(z) \in \mathbb{C}[[z]]^{\times n}$ and $o(H(z)) \geq 1$ is a quasi-translation if $j(F)(0) \neq 0$ and its formal inverse map is given by $G(z)=z+H(z)$.

Therefore, for any $P(z) \in \mathbb{C}[[z]]$ with $o(P(z)) \geq 2$ and $(\operatorname{Hes} P)(0)$ nilpotent, $P(z)$ is self-inverting iff the associated symmetric formal map $F(z)=z-\nabla P(z)$ is a quasi-translation.

The following general result has been proved in Proposition 1.1 of [B] for polynomial quasi-translations.

Proposition 4.2. A formal map $F(z)=z-H(z)$ with $o(H) \geq 1$ and $J H(0)$ nilpotent is a quasi-translation if and only if $J H \cdot H=0$.

The proof in $[\mathrm{B}]$ works equally well for formal quasi-translations under the condition that $J H(0)$ is nilpotent. Since it has also been shown in Proposition 1.1 of $[\mathrm{B}]$ that, for any polynomial quasi-translation $F(z)=z-H(z)$, $J H(z)$ is always nilpotent, the condition that $J H(0)$ is nilpotent in the proposition above does not put any extra restriction for the case of polynomial quasi-translations.

From Proposition 4.2, we immediately have the following criterion for self-inverting formal power series.

Proposition 4.3. For any $P(z) \in \mathbb{C}[[z]]$ with $o(P) \geq 2$ and $(\operatorname{Hes} P)(0)$ nilpotent, $P(z)$ is self-inverting if and only if $\langle\nabla P, \nabla P\rangle=0$.

Proof. Since $o(P) \geq 2$ and $(\operatorname{Hes} P)(0)$ is nilpotent, by Proposition 4.2 we know that $P(z)$ is self-inverting iff $J(\nabla P) \cdot \nabla P=(\operatorname{Hes} P) \cdot \nabla P=0$. But, 
on the other hand, it is easy to check that, for any $P(z) \in \mathbb{C}[[z]]$, we have the following identity:

$$
(\operatorname{Hes} P) \cdot \nabla P=\frac{1}{2} \nabla\langle\nabla P, \nabla P\rangle \text {. }
$$

Therefore, $($ Hes $P) \cdot \nabla P=0$ iff $\nabla\langle\nabla P, \nabla P\rangle=0$, and iff $\langle\nabla P, \nabla P\rangle=0$ because $o(\langle\nabla P, \nabla P\rangle) \geq 2$.

Corollary 4.4. For any $P(z) \in \mathbb{C}[[z]]$ with $o(P) \geq 2$ and $(\operatorname{Hes} P)(0)$ nilpotent, if $P(z)$ is self-inverting, then so is $P^{m}(z)$ for any $m \geq 1$.

Proof. For any $m \geq 2$, we have $o\left(P^{m}(z)\right) \geq 2 m>2$ and $(\operatorname{Hes} P)(0)=0$. Hence the corollary follows immediately from Proposition 4.3 and the following general identity:

$$
\left\langle\nabla P^{m}, \nabla P^{m}\right\rangle=m^{2} P^{2 m-2}\langle\nabla P, \nabla P\rangle
$$

Corollary 4.5. For any harmonic $P(z) \in \mathbb{C}[[z]]$ with $o(P) \geq 2$ and $($ Hes $P)(0)$ nilpotent, $P(z)$ is self-inverting iff $\Delta P^{2}=0$.

Proof. This follows immediately from Proposition 4.3 and the following general identity:

$$
\Delta P^{2}=2(\Delta P) P+2\langle\nabla P, \nabla P\rangle .
$$

Proposition 4.6. Let $P(z)$ be a harmonic self-inverting formal power series. Then, for any $m \geq 1, P^{m}$ is $H N$.

Proof. First, we use induction on $m \geq 1$ to show that $\Delta P^{m}=0$ for any $m \geq 1$.

The case of $m=1$ is our assumption. For any $m \geq 2$, consider

$$
\begin{aligned}
\Delta P^{m} & =\Delta\left(P \cdot P^{m-1}\right)=(\Delta P) P^{m-1}+P\left(\Delta P^{m-1}\right)+2\left\langle\nabla P, \nabla P^{m-1}\right\rangle \\
& =(\Delta P) P^{m-1}+P\left(\Delta P^{m-1}\right)+2(m-1) P^{m-2}\langle\nabla P, \nabla P\rangle .
\end{aligned}
$$

Then, by the induction assumption and Proposition 4.3 , we get $\Delta P^{m}=0$.

Secondly, for any fixed $m \geq 1$ and $d \geq 1$, we have

$$
\Delta^{d}\left[\left(P^{m}\right)^{d}\right]=\Delta^{d-1}\left(\Delta P^{d m}\right)=0 .
$$

Then, by the criterion in Proposition $1.2, P^{m}$ is $\mathrm{HN}$.

ExAMPLE 4.7. Note that, in Section 5.2 of [Z2], a family of self-inverting HN formal power series has been constructed as follows.

Let $\Xi$ be any non-empty subset of $\mathbb{C}^{n}$ such that, for any $\alpha, \beta \in \Xi$, $\langle\alpha, \beta\rangle=0$. Let $\mathcal{A}$ be the completion of the subalgebra of $\mathbb{C}[[z]]$ generated by $h_{\alpha}(z):=\langle\alpha, z\rangle(\alpha \in \Xi)$, i.e. $\mathcal{A}$ is the set of all formal power series in $h_{\alpha}(z)$ $(\alpha \in \Xi)$ over $\mathbb{C}$. Then it is straightforward to check (see Section 5.2 of [Z2] for details) that any $P(z) \in \mathcal{A}$ is $\mathrm{HN}$ and self-inverting. 
It is unknown if all HN self-inverting polynomials or formal power series can be obtained by the construction above. More generally, we believe the following open problem is worth investigating.

Open Problem 4.8.

(a) Decide whether or not all self-inverting polynomials or formal power series are $H N$.

(b) Classify all HN self-inverting polynomials and formal power series.

Finally, let us point out that, for any self-inverting $P(z) \in \mathbb{C}[[z]]$, the deformed inversion pair $Q_{t}(z)$ (not just $Q(z)=Q_{t=1}(z)$ ) is also $P(z)$.

Proposition 4.9. Let $P(z) \in \mathbb{C}[[z]]$ with $o(P) \geq 2$ and $(\operatorname{Hes} P)(0)$ nilpotent. Then $P(z)$ is self-inverting if and only if $Q_{t}(z)=P(z)$.

Proof. First, let us make the following observations.

Let $t$ be a formal central parameter and $F_{t}(z)=z-t \nabla P(z)$ as before. Since $o(P) \geq 2$ and $($ Hes $P)(0)$ is nilpotent, we have $j\left(F_{t}\right)(0)=1$. Therefore, $F_{t}(z)$ is an automorphism of the algebra $\mathbb{C}[t][[z]]$ of formal power series of $z$ over $\mathbb{C}[t]$. Since the inverse map of $F_{t}(z)$ is given by $G_{t}(z)=z+t \nabla Q_{t}(z)$, we see that $Q_{t}(z) \in \mathbb{C}[t][[z]]$. Therefore, for any $t_{0} \in \mathbb{C}, Q_{t=t_{0}}(z)$ makes sense and lies in $\mathbb{C}[[z]]$. Furthermore, by the uniqueness of inverse maps, it is easy to see that the inverse map of $F_{t_{0}}=z-t_{0} \nabla P$ of $\mathbb{C}[t][[z]]$ is given by $G_{t_{0}}(z)=z+t_{0} \nabla Q_{t=t_{0}}$. Therefore the inversion pair of $t_{0} P(z)$ is $t_{0} Q_{t=t_{0}}(z)$.

With the notation and observations above, by choosing $t_{0}=1$, we have $Q_{t=1}(z)=Q(z)$, and the $(\Leftarrow)$ part of the proposition follows immediately. Conversely, for any $t_{0} \in \mathbb{C}$, we have $\left\langle\nabla\left(t_{0} P\right), \nabla\left(t_{0} P\right)\right\rangle=t_{0}^{2}\langle\nabla P, \nabla P\rangle$. Then, by Proposition 4.3, $t_{0} P(z)$ is self-inverting and its inversion pair $t_{0} Q_{t=t_{0}}(z)$ is $t_{0} P(z)$. Therefore, $Q_{t=t_{0}}(z)=P(z)$ for any $t_{0} \in \mathbb{C}^{\times}$. But on the other hand, $Q_{t}(z) \in \mathbb{C}[t][[z]]$ as pointed out above, i.e. the coefficients of all monomials of $z$ in $Q_{t}(z)$ are polynomials of $t$, hence we must have $Q_{t}(z)=P(z)$, which is the $(\Rightarrow)$ part of the proposition.

\section{The vanishing conjecture over fields of positive characteris-} tic. It is well-known that the JC may fail when $F(z)$ is not a polynomial map (e.g. $\left.F_{1}\left(z_{1}, z_{2}\right)=e^{-z_{1}}, F_{2}\left(z_{1}, z_{2}\right)=z_{2} e^{z_{1}}\right)$. It also fails badly over fields of positive characteristic even in the one variable case (e.g. $F(x)=x-x^{p}$ over a field of characteristic $p>0$ ). However, the situation for the VC over fields of positive characteristic is dramatically different from the JC even though these two conjectures are equivalent to each other over fields of characteristic zero. Actually, as we will show in the proposition below, the $\mathrm{VC}$ over fields of positive characteristic holds for any polynomials (not even necessarily $\mathrm{HN}$ ) and also for any $\mathrm{HN}$ formal power series. 
Proposition 5.1. Let $k$ be a field of characteristic $p>0$. Then:

(a) For any polynomial $P(z) \in k[z]$ (not necessarily homogeneous nor $H N)$ of degree $d \geq 1, \Delta^{m} P^{m+1}=0$ for any $m \geq d(p-1) / 2$.

(b) For any $H N$ formal power series $P(z) \in k[[z]]$, i.e. $\Delta^{m} P^{m}=0$ for any $m \geq 1$, we have $\Delta^{m} P^{m+1}=0$ for any $m \geq p-1$.

In other words, over fields of positive characteristic, the VC holds even for HN formal power series $P(z) \in k[[z]]$; while for polynomials, it holds even without the HN condition or any other conditions.

Proof. The main reason that the proposition above holds is because of the following simple fact due to the Leibniz rule and positivity of the characteristic of the base field $k$ : for $m \geq 1, u(z), v(z) \in k[[z]]$ and any differential operator $\Lambda$ on $k[z]$, we have

$$
\Lambda\left(u^{m p} v\right)=u^{m p} \Lambda v
$$

Now let $P(z)$ be any polynomial or formal series as in the proposition. For any $m \geq 1$, write $m+1=q_{m} p+r_{m}$ with $q_{m}, r_{m} \in \mathbb{Z}$ and $0 \leq r_{m} \leq p-1$. Then by (5.1), we have

$$
\Delta^{m} P^{m+1}=\Delta^{m}\left(P^{q_{m} p} P^{r_{m}}\right)=P^{q_{m} p} \Delta^{m} P^{r_{m}} .
$$

If $P(z)$ is a polynomial of degree $d \geq 1$, we have $\Delta^{m} P^{r_{m}}=0$ when $m \geq d(p-1) / 2$, since in this case $2 m>\operatorname{deg}\left(P^{r_{m}}\right)$. If $P(z)$ is a HN formal power series, we have $\Delta^{m} P^{r_{m}}=0$ when $m \geq p-1 \geq r_{m}$. Therefore, (a) and (b) follow from (5.2) and the observations above.

An interesting question is whether or not the VC fails (as the JC does) for any $\mathrm{HN}$ formal power series $P(z) \in \mathbb{C}[[z]]$ but $P(z) \notin \mathbb{C}[z]$. To the best of our knowledge, no such counterexample has been given yet. We here state it as an open problem.

Open Problem 5.2. Find a $H N$ formal power series $P(z) \in \mathbb{C}[[z]]$ but $P(z) \notin \mathbb{C}[z]$, if any, such that the VC fails for $P(z)$.

Note that the crucial fact used in the proof of Proposition 5.1 is that any differential operator $\Lambda$ of $k[z]$ commutes with the multiplication by the $p$ th power of any element of $k[[z]]$. By a parallel argument, it is easy to see that the following more general result holds.

Proposition 5.3. Let $k$ be a field of characteristic $p>0$ and $\Lambda$ a differential operator on $k[z]$. Let $f \in k[[z]]$. Assume that, for any $1 \leq m \leq p-1$, there exists $N_{m}>0$ such that $\Lambda^{N_{m}} f^{m}=0$. Then $\Lambda^{m} f^{m+1}=0$ when $m \gg 0$.

In particular, if $\Lambda$ strictly decreases the degree of polynomials, then, for any polynomial $f \in k[z]$, we have $\Lambda^{m} f^{m+1}=0$ when $m \gg 0$. 


\section{A criterion of Hessian nilpotency for homogeneous polyno-} mials. Recall that $\langle\cdot, \cdot\rangle$ denotes the standard $\mathbb{C}$-bilinear form on $\mathbb{C}^{n}$. For any $\beta \in \mathbb{C}^{n}$, we set $h_{\beta}(z):=\langle\beta, z\rangle$ and $\beta_{D}:=\langle\beta, D\rangle$.

The main result of this section is the following criterion of Hessian nilpotency for homogeneous polynomials. Considering the criterion given in Proposition 1.2, it is somewhat surprising but the proof turns out to be very simple.

Theorem 6.1. For any $\beta \in \mathbb{C}^{n}$ and homogeneous polynomial $P(z)$ of degree $d \geq 2$, set $P_{\beta}(z):=\beta_{D}^{d-2} P(z)$. Then

$$
\text { Hes } P_{\beta}=(d-2) !(\operatorname{Hes} P)(\beta) \text {. }
$$

In particular, $P(z)$ is $H N$ iff, for any $\beta \in \mathbb{C}^{n}, P_{\beta}(z)$ is $H N$.

To prove the theorem, we need first the following lemma.

Lemma 6.2. Let $\beta \in \mathbb{C}^{n}$ and $P(z) \in \mathbb{C}[z]$ homogeneous of degree $N \geq 1$. Then

$$
\beta_{D}^{N} P(z)=N ! P(\beta) .
$$

Proof. Since both sides of (6.2) are linear in $P(z)$, we may assume $P(z)$ is a monomial, say $P(z)=z^{\mathbf{a}}$ for some $\mathbf{a} \in \mathbb{N}^{n}$ with $|\mathbf{a}|=N$. Then

$$
\begin{aligned}
\beta_{D}^{N} P(z) & =\left(\sum_{i=1}^{n} \beta_{i} D_{i}\right)^{N} z^{\mathbf{a}}=\sum_{\substack{\mathbf{k} \in \mathbb{N}^{n} \\
|\mathbf{k}|=N}}^{n} \frac{N !}{\mathbf{k} !} \beta^{\mathbf{k}} D^{\mathbf{k}} z^{\mathbf{a}} \\
& =\frac{N !}{\mathbf{a} !} \beta^{\mathbf{a}} D^{\mathbf{a}} z^{\mathbf{a}}=N ! \beta^{\mathbf{a}}=N ! P(\beta) .
\end{aligned}
$$

Proof of Theorem 6.1. We have

$$
\text { Hes } P_{\beta}(z)=\left(\frac{\partial^{2}\left(\beta_{D}^{d-2} P\right)}{\partial z_{i} \partial z_{j}}(z)\right)_{n \times n}=\left(\beta_{D}^{d-2} \frac{\partial^{2} P}{\partial z_{i} \partial z_{j}}(z)\right)_{n \times n}
$$

(applying Lemma 6.2 to $\frac{\partial^{2} P}{\partial z_{i} \partial z_{j}}(z):$ )

$$
=(d-2) !\left(\frac{\partial^{2} P}{\partial z_{i} \partial z_{j}}(\beta)\right)_{n \times n}=(d-2) !(\operatorname{Hes} P)(\beta) .
$$

Let $\left\{e_{i} \mid 1 \leq i \leq n\right\}$ be the standard basis of $\mathbb{C}^{n}$. Applying the theorem above to $\beta=e_{i}(1 \leq i \leq n)$, we obtain the following corollary, which was first proved by M. Kumar $[\mathrm{K}]$.

Corollary 6.3. For any homogeneous $H N$ polynomial $P(z) \in \mathbb{C}[z]$ of degree $d \geq 2, D_{i}^{d-2} P(z)(1 \leq i \leq n)$ are also $H N$. 
The reason that we think the criteria given in Theorem 6.1 and Corollary 6.3 are interesting is that $P_{\beta}(z)=\beta_{D}^{d-2} P(z)$ is homogeneous of degree 2 , and it is much easier to decide whether a homogeneous polynomial of degree 2 is $\mathrm{HN}$ or not. More precisely, for any homogeneous polynomial $U(z)$ of degree 2 , there exists a unique symmetric $n \times n$ matrix $A$ such that $U(z)=z^{\tau} A z$. Then it is easy to check that Hes $U(z)=2 A$. Therefore, $U(z)$ is HN iff the symmetric matrix $A$ is nilpotent.

We end this section with an open question concerning the criterion given in Proposition 1.2. To state it, we need to sketch the proof, given in [Z2].

For any $m \geq 1$, we set

$$
\begin{aligned}
& u_{m}(P)=\operatorname{Tr} \operatorname{Hes}^{m}(P), \\
& v_{m}(P)=\Delta^{m} P^{m} .
\end{aligned}
$$

For any $k \geq 1$, we define $\mathcal{U}_{k}(P)\left(\right.$ resp. $\left.\mathcal{V}_{k}(P)\right)$ to be the ideal in $\mathbb{C}[[z]]$ generated by $\left\{u_{m}(P) \mid 1 \leq m \leq k\right\}$ (resp. $\left\{v_{m}(P) \mid 1 \leq m \leq k\right\}$ ) and all their partial derivatives of any order. Then it has been shown (in a more general setting) in Section 4 of [Z2] that $\mathcal{U}_{k}(P)=\mathcal{V}_{k}(P)$ for any $k \geq 1$.

It is well-known in linear algebra that if $u_{m}(P(z))=0$ when $m \gg 0$, then Hes $P$ is nilpotent and $u_{m}(P)=0$ for any $m \geq 1$. A natural question is whether or not this is also the case for the sequence $\left\{v_{m}(P) \mid m \geq 1\right\}$. More precisely, we believe the following conjecture proposed in $[\mathrm{Z} 2]$ is worth investigating.

Conjecture 6.4. Let $P(z) \in \mathbb{C}[[z]]$ with $o(P(z)) \geq 2$. If $\Delta^{m} P^{m}(z)=0$ for $m \gg 0$, then $P(z)$ is $H N$.

7. Some results on symmetric polynomial maps. Let $P(z)$ be any formal power series with $o(P(z)) \geq 2$ and $($ Hes $P)(0)$ nilpotent, and $F(z)$ and $G(z)$ as before. Set

$$
\begin{aligned}
\sigma_{2} & :=\sum_{i=1}^{n} z_{i}^{2}, \\
f(z) & :=\frac{1}{2} \sigma_{2}-P(z) .
\end{aligned}
$$

Mohan Kumar [K] and David Wright [Wr3] asked how to write $P(z)$ and $f(z)$ in terms of $F(z)$. More precisely, the problem is to find $U(z), V(z) \in \mathbb{C}[[z]]$ such that

$$
\begin{aligned}
U(F(z)) & =P(z), \\
V(F(z)) & =f(z) .
\end{aligned}
$$


In this section, we first derive in Proposition 7.2 some explicit formulas for $U(z)$ and $V(z)$, and also for $W(z) \in \mathbb{C}[[z]]$ such that

$$
W(F(z))=\sigma_{2}(z) .
$$

We then show in Theorem 7.4 that, when $P(z)$ is a $\mathrm{HN}$ polynomial, the VC holds for $P$ or equivalently, the JC holds for the associated symmetric polynomial map $F(z)=z-\nabla P$, iff one of $U, V, W$ is a polynomial.

Let $t$ be a central parameter and $F_{t}(z)=z-t \nabla P$. Let $G_{t}(z)=z+t \nabla Q_{t}$ be the formal inverse of $F_{t}(z)$ as before. We set

$$
\begin{aligned}
f_{t}(z) & :=\frac{1}{2} \sigma_{2}-t P(z), \\
U_{t}(z) & :=P\left(G_{t}(z)\right), \\
V_{t}(z) & :=f_{t}\left(G_{t}(z)\right), \\
W_{t}(z) & :=\sigma_{2}\left(G_{t}(z)\right) .
\end{aligned}
$$

Note first that, under the conditions that $o(P(z)) \geq 2$ and $(\operatorname{Hes} P)(0)$ is nilpotent, we have $G_{t}(z) \in \mathbb{C}[t][[z]]^{\times n}$ as mentioned in the proof of Proposition 4.9. Therefore, $U_{t}(z), V_{t}(z), W_{t}(z) \in \mathbb{C}[t][[z]]$, and $U_{t=1}(z), V_{t=1}(z)$ and $W_{t=1}(z)$ all make sense. Secondly, from the definitions above,

$$
\begin{aligned}
W_{t}(z) & =2 V_{t}(z)+2 t U_{t}(z), \\
F_{t}(z) & =\nabla f_{t}(z), \\
f_{t=1}(z) & =f(z) .
\end{aligned}
$$

Lemma 7.1. With the notations above, we have

$$
\begin{aligned}
P(z) & =U_{t=1}(F(z)), \\
f(z) & =V_{t=1}(F(z)), \\
\sigma_{2}(z) & =W_{t=1}(F(z)) .
\end{aligned}
$$

In particular, $f(z), P(z)$ and $\sigma_{2}(z)$ lie in $\mathbb{C}[F]$ iff $U_{t=1}(z), V_{t=1}(z)$ and $W_{t=1}(z)$ lie in $\mathbb{C}[z]$.

In other words, by setting $t=1, U_{t}, V_{t}$ and $W_{t}$ will give us $U, V$ and $W$ in (7.3)-(7.5), respectively.

Proof. From the definitions of $U_{t}(z), V_{t}(z)$ and $W_{t}(z)$ (see (7.7)-(7.9)), we have

$$
P(z)=U_{t}\left(F_{t}(z)\right), \quad f_{t}(z)=V_{t}\left(F_{t}(z)\right), \quad \sigma_{2}(z)=W_{t}\left(F_{t}(z)\right) .
$$

By setting $t=1$ in the equations above and noticing that $F_{t=1}(z)=F(z)$, we get (7.13)-(7.15).

For $U_{t}(z), V_{t}(z)$ and $W_{t}(z)$, we have the following explicit formulas in terms of the deformed inversion pair $Q_{t}$ of $P$. 
Proposition 7.2. For any formal power series $P(z) \in \mathbb{C}[[z]]$ (not necessarily $H N)$ with $o(P(z)) \geq 2$ and $(\operatorname{Hes} P)(0)$ nilpotent, we have

$$
\begin{aligned}
U_{t}(z) & =Q_{t}+t \frac{\partial Q_{t}}{\partial t}, \\
V_{t}(z) & =\frac{1}{2} \sigma_{2}+t\left(z \frac{\partial Q_{t}}{\partial z}-Q_{t}\right), \\
W_{t}(z) & =\sigma_{2}+2 t z \frac{\partial Q_{t}}{\partial z}+2 t^{2} \frac{\partial Q_{t}}{\partial t} .
\end{aligned}
$$

Proof. Note first that, (7.18) follows directly from (7.16), (7.17) and (7.10).

To show (7.16), by $[\mathrm{Z1},(3.4)$ and (3.6)], we have

$$
U_{t}(z)=P\left(G_{t}\right)=Q_{t}+\frac{t}{2}\left\langle\nabla Q_{t}, \nabla Q_{t}\right\rangle=Q_{t}+t \frac{\partial Q_{t}}{\partial t} .
$$

To show (7.17), we consider

$$
\begin{aligned}
V_{t}(z) & =f_{t}\left(G_{t}\right) \\
& =\frac{1}{2}\left\langle z+t \nabla Q_{t}(z), z+t \nabla Q_{t}(z)\right\rangle-t P\left(G_{t}\right) \\
& =\frac{1}{2} \sigma_{2}+t\left\langle z, \nabla Q_{t}(z)\right\rangle+\frac{t^{2}}{2}\left\langle\nabla Q_{t}, \nabla Q_{t}\right\rangle-t P\left(G_{t}\right)
\end{aligned}
$$

(by (7.19), substituting $Q_{t}+\frac{t}{2}\left\langle\nabla Q_{t}, \nabla Q_{t}\right\rangle$ for $P\left(G_{t}\right)$ :)

$$
\begin{aligned}
& =\frac{1}{2} \sigma_{2}+t\left\langle z, \nabla Q_{t}(z)\right\rangle-t Q_{t}(z) \\
& =\frac{1}{2} \sigma_{2}+t\left(z \frac{\partial Q_{t}}{\partial z}-Q_{t}\right)
\end{aligned}
$$

When $P(z)$ is homogeneous and HN, we have the following more explicit formulas which in particular give solutions to the questions raised by Mohan Kumar and David Wright.

Corollary 7.3. For any homogeneous HN polynomial $P(z)$ of degree $d \geq 2$, we have

$$
\begin{aligned}
U_{t}(z) & =\sum_{m=0}^{\infty} \frac{t^{m}}{2^{m}(m !)^{2}} \Delta^{m} P^{m+1}(z) \\
V_{t}(z) & =\frac{1}{2} \sigma_{2}+\sum_{m=0}^{\infty} \frac{\left(d_{m}-1\right) t^{m+1}}{2^{m} m !(m+1) !} \Delta^{m} P^{m+1}(z), \\
W_{t}(z) & =\sigma_{2}+\sum_{m=0}^{\infty} \frac{\left(d_{m}+m\right) t^{m+1}}{2^{m-1} m !(m+1) !} \Delta^{m} P^{m+1}(z),
\end{aligned}
$$

where $d_{m}=\operatorname{deg}\left(\Delta^{m} P^{m+1}\right)=d(m+1)-2 m(m \geq 0)$. 
Proof. We give a proof for (7.20). (7.21) can be proved similarly, and (7.22) follows directly from (7.20), (7.21) and (7.10).

By combining (7.16) and (1.1), we have

$$
\begin{aligned}
U_{t}(z) & =\sum_{m=0}^{\infty} \frac{t^{m} \Delta^{m} P^{m+1}(z)}{2^{m} m !(m+1) !}+\sum_{m=1}^{\infty} \frac{m t^{m} \Delta^{m} P^{m+1}(z)}{2^{m} m !(m+1) !} \\
& =P(z)+\sum_{m=1}^{\infty} \frac{t^{m}}{2^{m}(m !)^{2}} \Delta^{m} P^{m+1}(z)=\sum_{m=0}^{\infty} \frac{t^{m}}{2^{m}(m !)^{2}} \Delta^{m} P^{m+1}(z) .
\end{aligned}
$$

A consequence of the proposition above is the following result on symmetric polynomial maps.

THEOREM 7.4. For any HN polynomial $P(z)$ (not necessarily homogeneous) with $o(P) \geq 2$, the following statements are equivalent:

(1) The VC holds for $P(z)$.

(2) $P(z) \in \mathbb{C}[F]$.

(3) $f(z) \in \mathbb{C}[F]$.

(4) $\sigma_{2}(z) \in \mathbb{C}[F]$.

Note that the equivalence of (1) and (3) was first proved by Mohan Kumar $([\mathrm{K}])$ by a different method.

Proof. Note first that, by Lemma 7.1, it is enough to show that $\Delta^{m} P^{m+1}$ $=0$ when $m \gg 0$ iff one of $U_{t}(z), V_{t}(z), W_{t}(z)$ is a polynomial in $t$ with coefficients in $\mathbb{C}[z]$. Secondly, when $P(z)$ is homogeneous, the statement above follows directly from (7.20)-(7.22).

To show the general case, for any $m \geq 0$ and $M_{t}(z) \in \mathbb{C}[t][[z]]$, we denote by $\left[t^{m}\right]\left(M_{t}(z)\right)$ the coefficient of $t^{m}$ when we write $M_{t}(z)$ as a formal power series in $t$ with coefficients in $\mathbb{C}[[z]]$. Then, from (7.16)-(7.18) and (1.1), it is straightforward to check that the coefficients of $t^{m}(m \geq 1)$ in $U_{t}(z), V_{t}(z)$ and $W_{t}(z)$ are

$$
\begin{gathered}
{\left[t^{m}\right]\left(U_{t}(z)\right)=\frac{\Delta^{m} P^{m+1}}{2^{m}(m !)^{2}},} \\
{\left[t^{m}\right]\left(V_{t}(z)\right)=\frac{1}{2^{m-1}(m-1) ! m !}\left(z \frac{\partial}{\partial z}\left(\Delta^{m-1} P^{m}\right)-\Delta^{m-1} P^{m}\right),} \\
{\left[t^{m}\right]\left(W_{t}(z)\right)} \\
=\frac{1}{2^{m-2}(m-1) ! m !}\left(z \frac{\partial}{\partial z}\left(\Delta^{m-1} P^{m}\right)+(m-1) \Delta^{m-1} P^{m}\right) .
\end{gathered}
$$

From (7.23), we immediately have $(1) \Leftrightarrow(2)$. To show the equivalences $(1) \Leftrightarrow(3)$ and $(1) \Leftrightarrow(4)$, note first that $o(P) \geq 2$, so $o\left(\Delta^{m-1} P^{m}\right) \geq 2$ for any $m \geq 1$. On the other hand, for any $h(z) \in \mathbb{C}[z]$ with $o(h(z)) \geq 2$, 
we have $h(z)=0$ iff $\left(z \frac{\partial}{\partial z}-1\right) h(z)=0$ iff $\left(z \frac{\partial}{\partial z}+(m-1)\right) h(z)=0$ for some $m \geq 1$. This is simply because, for any monomial $z^{\alpha}\left(\alpha \in \mathbb{N}^{n}\right)$, we have $\left(z \frac{\partial}{\partial z}-1\right) z^{\alpha}=(|\alpha|-1) z^{\alpha}$ and $\left(z \frac{\partial}{\partial z}+(m-1)\right) z^{\alpha}=(|\alpha|+(m-1)) z^{\alpha}$. From this general fact, we see that $(1) \Leftrightarrow(3)$ follows from $(7.24)$, and $(1) \Leftrightarrow(4)$ from (7.25).

8. A graph associated with homogeneous HN polynomials. In this section, we would like to draw the reader's attention to a graph $\mathcal{G}(P)$ assigned to each homogeneous harmonic polynomials $P(z)$. The graph $\mathcal{G}(P)$ was first proposed by the author and later studied by R. Willems in his master thesis [Wi] under the direction of A. van den Essen. The introduction of the graph $\mathcal{G}(P)$ is mainly motivated by a criterion of Hessian nilpotency given in [Z2] (see also Theorem 8.2 below), via which one hopes more necessary or sufficient conditions for a homogeneous harmonic polynomial $P(z)$ to be $\mathrm{HN}$ can be obtained or described in terms of the graph structure of $\mathcal{G}(P)$.

We first give the definition of the graph $\mathcal{G}(P)$ for any homogeneous harmonic polynomial $P(z)$ and discuss the connectedness reduction (see Corollary 8.5), i.e. a reduction of the $\mathrm{VC}$ to the homogeneous $\mathrm{HN}$ polynomials $P$ such that $\mathcal{G}(P)$ is connected. We then consider a connection of $\mathcal{G}(P)$ with the tree expansion formula derived in $[\mathrm{M}]$ and $[\mathrm{Wr} 2]$ for the inversion pair $Q(z)$ of $P(z)$ (see Proposition 8.9). As an application, we give another proof for the connectedness reduction given in Corollary 8.5.

8.1. Definition and connectedness reduction. For any $\beta \in \mathbb{C}^{n}$, set $h_{\beta}(z)$ $:=\langle\beta, z\rangle$ and $\beta_{D}:=\langle\beta, D\rangle$, where $\langle\cdot, \cdot\rangle$ is the standard $\mathbb{C}$-bilinear form on $\mathbb{C}^{n}$. Let $X(\mathbb{C})$ denote the set of all isotropic elements of $\mathbb{C}^{n}$, i.e. of all $\alpha \in \mathbb{C}^{n}$ such that $\langle\alpha, \alpha\rangle=0$.

Recall that we have the following fundamental theorem on homogeneous harmonic polynomials.

TheOREM 8.1. For any homogeneous harmonic polynomial $P(z)$ of degree $d \geq 2$, we have

$$
P(z)=\sum_{i=1}^{k} c_{i} h_{\alpha_{i}}^{d}(z)
$$

for some $c_{i} \in \mathbb{C}^{\times}$and $\alpha_{i} \in X\left(\mathbb{C}^{n}\right)(1 \leq i \leq k)$.

Note that, replacing $\alpha_{i}$ in (8.1) by $c_{i}^{-1 / d} \alpha_{i}$, we may also write $P(z)$ as

$$
P(z)=\sum_{i=1}^{k} h_{\alpha_{i}}^{d}(z)
$$

with $\alpha_{i} \in X\left(\mathbb{C}^{n}\right)(1 \leq i \leq k)$.

For the proof of Theorem 8.1, see, for example, [I] and [Wi]. 
We fix a homogeneous harmonic polynomial $P(z) \in \mathbb{C}[z]$ of degree $d \geq 2$, and assume that $P(z)$ is given by $(8.2)$ for some $\alpha_{i} \in X\left(\mathbb{C}^{n}\right)(1 \leq i \leq k)$. We may and will always assume that $\left\{h_{\alpha_{i}}^{d}(z) \mid 1 \leq i \leq k\right\}$ are linearly independent in $\mathbb{C}[z]$.

Recall the following matrices introduced in [Z2]:

$$
\begin{aligned}
A_{P} & =\left(\left\langle\alpha_{i}, \alpha_{j}\right\rangle\right)_{k \times k}, \\
\Psi_{P} & =\left(\left\langle\alpha_{i}, \alpha_{j}\right\rangle h_{\alpha_{j}}^{d-2}(z)\right)_{k \times k} .
\end{aligned}
$$

Then we have the following criterion of Hessian nilpotency for homogeneous harmonic polynomials. For its proof, see Theorem 4.3 in [Z2].

TheOREM 8.2. Let $P(z)$ be as above. Then, for any $m \geq 1$, we have

$$
\operatorname{Tr} \operatorname{Hes}^{m}(P)=(d(d-1))^{m} \operatorname{Tr} \Psi_{P}^{m} .
$$

In particular, $P(z)$ is $H N$ if and only if the matrix $\Psi_{P}$ is nilpotent.

A simple remark on the criterion above is as follows.

Let $B$ be the $k \times k$ diagonal matrix with the $i$ th $(1 \leq i \leq k)$ diagonal entry being $h_{\alpha_{i}}(z)$. For any $1 \leq j \leq k$, set

$$
\Psi_{P ; j}:=B^{j} A_{P} B^{d-2-j}=\left(h_{\alpha_{i}}^{j}\left\langle\alpha_{i}, \alpha_{j}\right\rangle h_{\alpha_{j}}^{d-2-j}\right) .
$$

Then, by repeatedly applying the fact that, for any two $k \times k$ matrices $C$ and $D, C D$ is nilpotent iff so is $D C$, it is easy to see that Theorem 8.2 can also be restated as follows.

Corollary 8.3. Let $P(z)$ be given by (8.2) with $d \geq 2$. Then, for any $1 \leq j \leq d-2$ and $m \geq 1$, we have

$$
\operatorname{Tr} \operatorname{Hes}^{m}(P)=(d(d-1))^{m} \operatorname{Tr} \Psi_{P ; j}^{m} .
$$

In particular, $P(z)$ is $H N$ if and only if the matrix $\Psi_{P ; j}$ is nilpotent.

Note that, when $d$ is even, we may choose $j=(d-2) / 2$. So $P$ is HN iff the symmetric matrix

$$
\Psi_{P ;(d-2) / 2}(z)=\left(h_{\alpha_{i}}^{(d-2) / 2}(z)\left\langle\alpha_{i}, \alpha_{j}\right\rangle h_{\alpha_{j}}^{(d-2) / 2}(z)\right)
$$

is nilpotent.

Motivated by the criterion above, we assign a graph $\mathcal{G}(P)$ to any homogeneous harmonic polynomial $P(z)$ as follows.

We fix an expression as in (8.2) for $P(z)$. The set of vertices of $\mathcal{G}(P)$ is the set of positive integers $[\mathbf{k}]:=\{1, \ldots, k\}$. The vertices $i$ and $j$ of $\mathcal{G}(P)$ are connected by an edge iff $\left\langle\alpha_{i}, \alpha_{j}\right\rangle \neq 0$. We thus get a finite graph.

Furthermore, we may also label edges of $\mathcal{G}(P)$ by assigning $\left\langle\alpha_{i}, \alpha_{j}\right\rangle$ or $\left(h_{\alpha_{i}}^{(d-2) / 2}\left\langle\alpha_{i}, \alpha_{j}\right\rangle h_{\alpha_{i}}^{(d-2) / 2}\right)$, when $d$ is even, to the edge connecting vertices $i, j \in[\mathbf{k}]$. We then get a labeled graph whose adjacency matrix is exactly $A_{P}$ or $\Psi_{P,(d-2) / 2}$ (depending on the labels we choose for the edges of $\mathcal{G}(P)$ ).

Naturally, one may also ask the following (open) questions. 
Open Problem 8.4.

(a) Find necessary or sufficient conditions on the (labeled) graph $\mathcal{G}(P)$ such that the homogeneous harmonic polynomial $P(z)$ is $H N$.

(b) Find necessary or sufficient conditions on the (labeled) graph $\mathcal{G}(P)$ such that the VC holds for the homogeneous HN polynomial $P(z)$.

First, let us point out that, to approach the open problems above, it is enough to focus on homogeneous harmonic polynomials $P$ such that the graph $\mathcal{G}(P)$ is connected.

Indeed, suppose that $\mathcal{G}(P)$ has $r \geq 2$ connected components. Let $[\mathbf{k}]=$ $\bigsqcup_{i=1}^{r} I_{i}$ be the corresponding partition of the set of vertices. For each $1 \leq$ $i \leq r$, we set $P_{i}(z):=\sum_{\alpha \in I_{i}} h_{\alpha}^{d}(z)$.

Note that, by Lemma 2.6 , the polynomials $P_{i}(1 \leq i \leq r)$ are pairwise disjoint, so Corollary 2.8 applies to the sum $P=\sum_{i=1}^{r} P_{i}$. In particular, we have,

(a) $P$ is $H N$ iff each $P_{i}$ is $H N$.

(b) if the VCholds for each $P_{i}$, then it also holds for $P$.

Therefore, we have the following connectedness reduction.

Corollary 8.5. To study homogeneous HN polynomials $P$ or the $V C$ for homogeneous $H N$ polynomials $P$, it is enough to consider the case when $\mathcal{G}(P)$ is connected.

Note that property (a) above was first proved by R. Willems ([Wi]) by using the criterion in Theorem 8.2. (b) had been proved by the author by a different argument, and with the author's permission, it was also included in $[\mathrm{Wi}]$.

Finally, let us point out that R. Willems ([Wi]) has proved the following very interesting results on Open Problem 8.4.

TheOREM $8.6([\mathrm{Wi}])$. Let $P$ be a homogeneous $H N$ polynomial as in (8.2) with $d \geq 4$. Let $l(P)$ be the dimension of the vector subspace of $\mathbb{C}^{n}$ spanned by $\left\{\alpha_{i} \mid 1 \leq i \leq k\right\}$. Then

(1) If $l(P)=1,2, k-1$ or $k$, then the graph $\mathcal{G}(P)$ is totally disconnected (i.e. it has no edges).

(2) If $l(P)=k-2$ and $\mathcal{G}(P)$ is connected, then $\mathcal{G}(P)$ is the complete bi-graph $K(4, k-4)$.

(3) In the case of (1) and (2) above, the VC holds.

Furthermore, it has also been shown in [Wi] that, for any homogeneous HN polynomial $P$, the graph $\mathcal{G}(P)$ cannot be a path or cycle of any positive length. For more details, see [Wi]. 
8.2. Connection with the tree expansion formula for inversion pairs. First let us recall the tree expansion formula derived in $[\mathrm{M}]$, [Wr2] for the inversion pair $Q(z)$.

Let $\mathbb{T}$ denote the set of all trees, i.e. of all connected and simply connected finite simple graphs. For each tree $T \in \mathbb{T}$, denote by $V(T)$ and $E(T)$ the sets of all vertices and edges of $T$, respectively. Then we have the following tree expansion formula for inversion pairs.

Theorem $8.7([\mathrm{M}],[\mathrm{Wr} 2])$. Let $P \in \mathbb{C}[[z]]$ with $o(P) \geq 2$ and $Q$ its inversion pair. For any $T \in \mathbb{T}$, set

$$
Q_{T, P}=\sum_{\ell: E(T) \rightarrow[\mathbf{n}]} \prod_{v \in V(T)} D_{\operatorname{adj}(v), \ell} P,
$$

where $\operatorname{adj}(v)$ is the set $\left\{e_{1}, \ldots, e_{s}\right\}$ of edges of $T$ adjacent to $v$, and $D_{\operatorname{adj}(v), \ell}$ $=D_{\ell\left(e_{1}\right)} \cdots D_{\ell\left(e_{s}\right)}$. Then the inversion pair $Q$ of $P$ is given by

$$
Q=\sum_{T \in \mathbb{T}} \frac{1}{|\operatorname{Aut}(T)|} Q_{T, P}
$$

Now we assume $P(z)$ is a homogeneous harmonic polynomial $d \geq 2$ of the form (8.2). It is easy to see that then

$$
Q_{T, P}=\sum_{f: V(T) \rightarrow[\mathbf{k}] \ell: E(T) \rightarrow[\mathbf{n}]} \prod_{v \in V(T)} D_{\operatorname{adj}(v), \ell} h_{\alpha_{f(v)}}^{d}(z) .
$$

The role played by the graph $\mathcal{G}(P)$ is to restrict the maps $f: V(T) \rightarrow$ $V(\mathcal{G}(P))(=[\mathbf{k}])$ in (8.11) to a special family of maps. To be more precise, let $\Omega(T, \mathcal{G}(P))$ be the set of maps $f: V(T) \rightarrow[\mathbf{k}]$ such that, for any distinct adjacent vertices $u, v \in V(T), f(u)$ and $f(v)$ are distinct and adjacent in $\mathcal{G}(P)$. Then we have the following lemma.

Lemma 8.8. For any $f: V(T) \rightarrow[\mathbf{k}]$ with $f \notin \Omega(T, \mathcal{G}(P))$, we have

$$
\sum_{\ell: E(T) \rightarrow[\mathbf{n}]} \prod_{v \in V(T)} D_{\operatorname{adj}(v), \ell} h_{\alpha_{f(v)}}^{d}(z)=0 .
$$

Proof. Let $f: V(T) \rightarrow[\mathbf{k}]$ as in the lemma. Since $f \notin \Omega(T, \mathcal{G}(P))$, there exist distinct adjacent $v_{1}, v_{2} \in V(T)$ such that either $f\left(v_{1}\right)=f\left(v_{2}\right)$ or $f\left(v_{1}\right)$ and $f\left(v_{2}\right)$ are not adjacent in the graph $\mathcal{G}(P)$. In any case, we have $\left\langle\alpha_{f\left(v_{1}\right)}, \alpha_{f\left(v_{2}\right)}\right\rangle=0$.

Next we consider the contributions to the RHS of (8.11) from the vertices $v_{1}$ and $v_{2}$. Denote by $e$ the edge of $T$ connecting $v_{1}$ and $v_{2}$, and $\left\{e_{1}, \ldots, e_{r}\right\}$ (resp. $\left\{\widetilde{e}_{1}, \ldots, \widetilde{e}_{s}\right\}$ ) the set of edges connected with $v_{1}$ (resp. $v_{2}$ ) besides the edge $e$. Then, for any $\ell: E(T) \rightarrow[\mathbf{n}]$, the factor in the RHS of (8.11) coming from the vertices $v_{1}$ and $v_{2}$ is the product

$$
\left(D_{\ell(e)} D_{\ell\left(e_{1}\right)} \cdots D_{\ell\left(e_{r}\right)} h_{\alpha_{f\left(v_{1}\right)}}^{d}(z)\right)\left(D_{\ell(e)} D_{\ell\left(\widetilde{e}_{1}\right)} \cdots D_{\ell\left(\widetilde{e}_{s}\right)} h_{\alpha_{f\left(v_{2}\right)}}^{d}(z)\right) .
$$


Define an equivalence relation for maps $\ell: E(T) \rightarrow[\mathbf{n}]$ by setting $\ell_{1} \sim \ell_{2}$ iff $\ell_{1}, \ell_{2}$ have the same value at each edge of $T$ except $e$. Then, by summing of the terms in (8.13) over each equivalence class, we get the factor

$$
\left\langle\nabla D_{\ell\left(e_{1}\right)} \cdots D_{\ell\left(e_{r}\right)} h_{\alpha_{f\left(v_{1}\right)}^{d}}^{d}(z), \nabla D_{\ell\left(\widetilde{e}_{1}\right)} \cdots D_{\ell\left(\widetilde{e}_{s}\right)} h_{\alpha_{f\left(v_{2}\right)}}^{d}(z)\right\rangle .
$$

Note that $D_{\ell\left(e_{1}\right)} \cdots D_{\ell\left(e_{r}\right)} h_{\alpha_{f\left(v_{1}\right)}}^{d}(z)$ and $D_{\ell\left(\widetilde{e}_{1}\right)} \cdots D_{\ell\left(\widetilde{e}_{s}\right)} h_{\alpha_{f\left(v_{2}\right)}}^{d}(z)$ are constant multiples of some integral powers of $h_{\alpha_{f\left(v_{1}\right)}}(z)$ and $h_{\alpha_{f\left(v_{2}\right)}}(z)$, respectively. Therefore, $\left\langle\alpha_{f\left(v_{1}\right)}, \alpha_{f\left(v_{2}\right)}\right\rangle(=0)$ appears as a multiplicative constant factor in the term in (8.14), which makes the term zero. Hence the lemma follows.

An immediate consequence of the lemma above is the following proposition.

Proposition 8.9. With the setting and notation as above, we have

$$
Q_{T, P}=\sum_{f \in \Omega(T, \mathcal{G}(P))} \sum_{\ell: E(T) \rightarrow[\mathbf{n}]} \prod_{v \in V(T)} D_{\operatorname{adj}(v), \ell} h_{\alpha_{f(v)}}^{d}(z) .
$$

REMARK 8.10.

(a) For any $f \in \Omega(T, \mathcal{G}(P)),\left\{f^{-1}(j) \mid j \in \operatorname{Im}(f)\right\}$ gives a partition of $V(T)$ since no two distinct vertices in $f^{-1}(j)(j \in \operatorname{Im}(f))$ can be adjacent. In other words, $f$ is nothing but a proper coloring for the tree $T$, which is also subject to certain more conditions from the graph structure of $\mathcal{G}(P)$. It is interesting to see that the coloring problem for graphs also plays a role in the inversion problem for symmetric formal maps.

(b) It would be interesting to derive more results from the graph $\mathcal{G}(P)$ via the formulas in (8.10) and (8.15).

REMARK 8.11. By similar arguments to those in the proof of Lemma 8.8, one can get another proof for Theorem 2.7 in the setting of Lemma 2.6.

Finally, as an application of Proposition 8.9 above, we give another proof for the connectedness reduction given in Corollary 8.5.

Let $P$ be as in (8.2) with inversion pair $Q$. Suppose that there exists a partition $[\mathbf{k}]=I_{1} \sqcup I_{2}$ with $I_{i} \neq \emptyset$. Let $P_{i}=\sum_{\alpha \in I_{i}} h_{\alpha}^{d}(z)(i=1,2)$ and $Q_{i}$ the inversion pair of $P_{i}$. Then $P=P_{1}+P_{2}$ and $\mathcal{G}\left(P_{1}\right) \sqcup \mathcal{G}\left(P_{2}\right)=\mathcal{G}(P)$. Therefore, to prove the connectedness reduction, it is enough to show $Q=Q_{1}+Q_{2}$. But this follows immediately from (8.10), (8.15) and the following lemma.

Lemma 8.12. Let $P, P_{1}$ and $P_{2}$ be as above. Then, for any tree $T \in \mathbb{T}$, we have

$$
\Omega(T, \mathcal{G}(P))=\Omega\left(T, \mathcal{G}\left(P_{1}\right)\right) \sqcup \Omega\left(T, \mathcal{G}\left(P_{2}\right)\right)
$$


Proof. For any $f \in \Omega(T, \mathcal{G}(P)), f$ preserves the adjacency of vertices of $\mathcal{G}(P)$. Since $T$ is a connected graph, so is $\operatorname{Im}(f) \subset V(\mathcal{G}(P))$, being a (full) subgraph of $\mathcal{G}(P)$. Therefore, $\operatorname{Im}(f) \subset V\left(\mathcal{G}\left(P_{1}\right)\right)$ or $\operatorname{Im}(f) \subset V\left(\mathcal{G}\left(P_{2}\right)\right)$. Hence $\Omega(T, \mathcal{G}(P)) \subset \Omega\left(T, \mathcal{G}\left(P_{1}\right)\right) \sqcup \Omega\left(T, \mathcal{G}\left(P_{2}\right)\right)$. The other inclusion is obvious.

\section{References}

[BCW] H. Bass, E. Connell and D. Wright, The Jacobian conjecture. Reduction of degree and formal expansion of the inverse, Bull. Amer. Math. Soc. 7 (1982), 287-330.

[B] M. de Bondt, Quasi-translations and counterexamples to the homogeneous dependence problem, Proc. Amer. Math. Soc. 134 (2006), 2849-2856.

[BE1] M. de Bondt and A. van den Essen, A reduction of the Jacobian conjecture to the symmetric case, ibid. 133 (2005), 2201-2205.

[BE2] - - - Nilpotent symmetric Jacobian matrices and the Jacobian conjecture, J. Pure Appl. Algebra 193 (2004), 61-70.

[BE3] -, 一, Singular Hessians, J. Algebra 282 (2004), 195-204.

[BE4] - - - Nilpotent symmetric Jacobian matrices and the Jacobian conjecture II, J. Pure Appl. Algebra 196 (2005), 135-148.

[BE5] -, - Hesse and the Jacobian conjecture, in: Affine Algebraic Geometry, Contemp. Math. 369, Amer. Math. Soc., Providence, RI, 2005, 63-76.

[E] A. van den Essen, Polynomial Automorphisms and the Jacobian Conjecture, Progr. Math. 190, Birkhäuser, Basel, 2000.

[EW] A. van den Essen and S. Washburn, The Jacobian conjecture for symmetric Jacobian matrices, J. Pure Appl. Algebra 189 (2004), 123-133.

[EZ] A. van den Essen and W. Zhao, Two results on Hessian nilpotent polynomials, J. Pure Appl. Algebra, to appear; arXiv:0704.1690v1 [math.AG].

[I] H. Iwaniec, Topics in Classical Automorphic Forms, Grad. Stud. in Math. 17, Amer. Math. Soc., Providence, RI, 1997.

[Ke] O. H. Keller, Ganze Cremona-Transformationen, Monatsh. Math. Phys. 47 (1939), 299-306.

[K] M. Kumar, personal communications.

[M] G. Meng, Legendre transform, Hessian conjecture and tree formula, Appl. Math. Lett. 19 (2006), 503-510; see also math-ph/0308035.

[R] R. M. Range, Holomorphic Functions and Integral Representations in Several Complex Variables, Grad. Texts in Math. 108, Springer, New York, 1986.

[Wa] S. Wang, A Jacobian criterion for separability, J. Algebra 65 (1980), 453-494.

[Wi] R. Willems, Graphs and the Jacobian conjecture, Master Thesis, Radboud Univ. Nijmegen, 2005.

[Wr1] D. Wright, The Jacobian conjecture: ideal membership questions and recent advances, in: Affine Algebraic Geometry, Contemp. Math. 369, Amer. Math. Soc., Providence, RI, 2005, 261-276.

[Wr2] - The Jacobian conjecture as a combinatorial problem, in: Affine Algebraic Geometry, Osaka Univ. Press, Osaka, 2007, 483-503; see also math.CO/0511214.

[Wr3] - personal communications.

[Y] A. V. Yagzhev, On a problem of O.-H. Keller, Sibirsk. Mat. Zh. 21 (1980), 141-150, 191 (in Russian). 
[Z1] W. Zhao, Inversion problem, Legendre transform and inviscid Burgers' equation, J. Pure Appl. Algebra 199 (2005), 299-317; see also math.CV/0403020.

[Z2] - Hessian nilpotent polynomials and the Jacobian conjecture, Trans. Amer. Math. Soc. 359 (2007), 249-274; see also math.CV/0409534.

Department of Mathematics

Illinois State University

Normal, IL 61790-4520, U.S.A.

E-mail: wzhao@ilstu.edu

Received 25.4.2007

and in final form 27.1.2008 